\title{
Better not to deal with two tasks at the same time when stressed? Acute psychosocial stress reduces task shielding in dual-task performance
}

\author{
Franziska Plessow • Susann Schade • \\ Clemens Kirschbaum • Rico Fischer
}

Published online: 10 June 2012

(C) Psychonomic Society, Inc. 2012

\begin{abstract}
A major control demand in successful dual-task performance is the task-specific separation of task-goal representations and of the related stimulus-response translation processes. In the present study, we investigated how these cognitive control processes of task shielding are affected by acute psychosocial stress. Fifty-six healthy participants were exposed to either an acute psychosocial stressor (the Trier Social Stress Test) or a standardized control situation prior to a dual task. Task shielding was assessed by analyzing the interference of Task 2 processing on prioritized Task 1 performance. Following successful stress induction, as indicated by increases in salivary $\alpha$-amylase (sAA) and cortisol that reflect increases in sympathetic nervous system and hypothalamus-pituitary-adrenal (HPA) axis activity, respectively, stressed individuals displayed reduced task shielding relative to controls. This result was further substantiated by a correlation between treatment-related increase in cortisol, but not sAA, and between-task interference, suggesting a potential role of the HPA stress response for the development of the observed effects. As an additional finding, when the volunteers were categorized with regard to their action-state orientation, their orientation did not interact with stress but did reveal generally increased between-task interference, and thus inferior task shielding, for state-oriented as compared to action-oriented individuals.
\end{abstract}

Electronic supplementary material The online version of this article (doi:10.3758/s13415-012-0098-6) contains supplementary material, which is available to authorized users.

F. Plessow $(\bowtie) \cdot$ S. Schade $\cdot$ C. Kirschbaum $\cdot$ R. Fischer Department of Psychology, Technische Universität Dresden, 01062 Dresden, Germany

e-mail: plessow@biopsych.tu-dresden.de
Keywords Executive functions - Cognitive control · Shielding · Dual task · Crosstalk · Acute psychosocial stress · Trier Social Stress Test · HPA axis · Cortisol

Technical progress during the last decades, particularly in the domains of communication technology and human-machine interactions, has tremendously increased dual-tasking requirements for the individual with regard to both quantity and quality. Similar to dual-tasking, stress has become an omnipresent aspect of modern life, and thus successful performing even under conditions of acute stress represents an everyday demand for more and more individuals. As a consequence, these similar trends are not only indicative of a need to address both issues, but also require an investigation of the specific link between acute stress and dual-task performance; this topic is pursued in the present study.

Whenever more than one task is performed at a time, the cognitive system faces additional demands that go beyond those of single-task processing (e.g., online order control, task-set separation, and task-component scheduling). Accordingly, theoretical models of dual-task performance (e.g., Logan \& Gordon, 2001; Meyer \& Kieras, 1997; Sigman \& Dehaene, 2006) assume that simultaneous task performance is coordinated and realized by mechanisms of cognitive control ${ }^{1}$

\footnotetext{
${ }^{1}$ It should be noted that this view is not shared by traditional dual-task models. For example, the influential response-selection bottleneck model holds that, due to a structural processing limitation, only certain information processing in two tasks can occur at the same time (e.g., perception and motor execution), whereas other critical stages (e.g., response selection) need to be performed one at a time (i.e., serially), resulting in a so-called psychological refractory period (see Pashler, 1998, for an overview). Importantly, information processing at the bottleneck is passively scheduled on a first come-first served basis, without the involvement of active control processes (for further elaboration, see the Discussion section).
} 
and crucially depends on the efficient allocation of attentional resources (Navon \& Miller, 2002; Tombu \& Jolicœur, 2003). The strategic-response-deferment model (Meyer \& Kieras, 1997; see also Logan \& Gordon, 2001), for example, holds that secondary task processing is strategically delayed in order to meet the attentional requirements of the prioritized primary task processing. More specifically, dual-task performance depends, for example, on the ability to protect prioritized task processing from the interfering influences (i.e., crosstalk) of secondary task processing in order to avoid performance errors that not only impair the task at hand, but most likely would deteriorate performance within the entire dual-task context. Therefore, task shielding reflects cognitive control processes that enable the reduction of between-task interference by a task-specific separation of task-goal representations and the related stimulus-response (S-R) translation processes (e.g., Logan \& Gordon, 2001)-for example, by increasing the activation of the prioritized task processing (Stelzel, Brandt, \& Schubert, 2009) and/or by inhibiting competing task(-component) processes (see Koch, Gade, Schuch, \& Philipp, 2010, for a review). Task shielding is of especially vital importance when both tasks are similar, and thus often represents a major challenge. It is therefore important to gain knowledge about how and which potential critical or beneficial factors jeopardize or determine the success of task shielding in dual-task performance. According to this reasoning, in the present study, we particularly focused on the question of how these cognitive control processes of task shielding are affected by acute stress experience.

The links between acute stress and cognitive control processes in dual-task performance are manifold. For example, early work in both laboratory and real-life settings has already suggested that prototypical dual-task demands (i.e., controlling an airplane) are related to increases in biological stress markers (e.g., Hale, Ellis, \& Kratochvil, 1959; Pincus $\&$ Hoagland, 1943). In addition, the demands of performing two or more tasks at the same time represent high levels of task complexity - a factor that was found to also increase the probability that stress would reveal effects on cognitive performance (e.g., Oei, Everaerd, Elzinga, van Well, \& Bermond, 2006; Schoofs, Wolf, \& Smeets, 2009). Most importantly, both the cognitive control processes of task shielding in the service of successful dual-task performance and the physiological effects of acute stress share substantial neural commonalities, such as their relation to the prefrontal cortex (PFC). Regarding dual-task performance, neuroimaging studies have pointed to involvement of both the (dorso) lateral (e.g., Dux, Ivanoff, Asplund, \& Marois, 2006; Marois \& Ivanoff, 2005; Stelzel et al., 2009) and rostral (e.g., Koechlin, Basso, Pietrini, Panzer, \& Grafman, 1999) PFC. Stelzel et al., for example, demonstrated that the dorsolateral PFC critically mediates shielding of the prioritized Task 1 (T1) by increasing activity in T1-relevant sensory areas, especially in conditions of simultaneous secondary Task 2 (T2) processing. At the same time, not only the cognitive control processes involved in dual-task performance, but also physiological stress effects, have been located in the PFC. When one is exposed to a stressor, two major stress axes get activated. First, stress induces an increase in sympathetic nervous system (SNS) activity and catecholamine release. High catecholamine levels have been found to decrease neuron firing rates in the PFC (cf. Ramos \& Arnsten, 2007; Vijayraghavan, Wang, Birnbaum, Williams, \& Arnsten, 2007). Second, and more slowly, stress triggers an activity increase in the hypothalamus-pituitary-adrenal (HPA) axis, which, in turn, induces the synthesis and release of glucocorticoids (primarily cortisol) into the bloodstream (e.g., de Kloet, Joels, \& Holsboer, 2005). Under conditions of elevated glucocorticoid levels (e.g., stress), glucocorticoids primarily bind to glucocorticoid receptors (e.g., de Kloet \& Reul, 1987) that are highly prevalent in the PFC (e.g., Perlman, Webster, Herman, Kleinman, \& Weickert, 2007; Sanchez, Young, Plotsky, \& Insel, 2000). Moreover, elevated glucocorticoid levels in conditions of acute stress have been found to alter PFC activity (e.g., Kern et al., 2008; Qin, Hermans, van Marle, Luo, \& Fernandez, 2009).

Despite these established links on the physiological level, direct empirical evidence of how the specific PFC-related cognitive control functions involved in dual-tasking are influenced by acute stress is still missing. To our knowledge, only a few studies have demonstrated an impact of acute stress on the flexible implementation of task goals that enables a switch between tasks when environmental changes demand behavioral adaptation (e.g., Liston, McEwen, \& Casey, 2009; Plessow, Kiesel, \& Kirschbaum, 2012). ${ }^{2}$ A recent study from our laboratory investigated the impact of acute stress on task (-goal) shielding in a selective-attention (i.e., Simon) task involving strong response conflict (Plessow, Fischer, Kirschbaum, \& Goschke, 2011). The measure of interest was the trial-to-trial sequential adjustment of task shielding related to an encountered response conflict. The results revealed that, relative to controls, stressed individuals displayed tonically increased task shielding that was not only present in situations in which task shielding was required and functional (i.e., following conflict trials), but also occurred in situations in which task shielding was not indicated (i.e., following nonconflict trials). Put differently, this increased task shielding came at the cost of reduced cognitive flexibility in selectively adjusting the amount of shielding to varying trial-to-trial task requirements. Although this tonic increase in task shielding was interpreted as a compensatory mechanism in response to acute stress, to date, it remains unclear whether increases in task shielding represent the default compensatory

\footnotetext{
${ }^{2}$ For a more general discussion of acute stress effects on PFCdependent cognitive processes, see the Discussion section.
} 
mechanism that is the "natural" response of the cognitive system to an acute stress experience (see also Easterbrook's cue-utilization hypothesis; Easterbrook, 1959) or whether stress might trigger a specific compensatory mechanism that entails the most economical resource demands in terms of "choosing" the most resource-efficient task-processing mode available. Indeed, in the selective-attention task of Plessow et al. (2011), the most parsimonious and resource-efficient task-processing strategy might entail a tonic blocking of task-irrelevant information in order to prevent interference, whereas a flexible trial-to-trial adjustment of task shielding to varying task demands (i.e., response conflict vs. no response conflict) might appear too risky, error-prone, and resource-consuming.

Investigating the effects of acute stress on task shielding in dual-task performance allowed us to directly test these two predictions. Even though dual-task research has provided evidence that a task-processing mode of strong task shielding represents the most favorable processing strategy (Logan \& Gordon, 2001; Miller, Ulrich, \& Rolke, 2009; Navon \& Miller, 1987), as it minimizes between-task interference (Koch, 2009), this mode is also associated with increased mental effort, since it requires temporary inhibition of $\mathrm{T} 2$ processing that has to be resumed as soon as $\mathrm{T} 1$ conductance is finished (Lehle, Steinhauser, \& Hübner, 2009). Accordingly, participants had rated conditions of strong task shielding as more demanding, had displayed an increased heart rate (a physiological marker of mental effort) as compared to conditions of weaker task shielding (Lehle et al., 2009), and had adopted a strategy of little task shielding when provided with free choice (Lehle \& Hübner, 2009).

On this basis, in the present study, the following predictions were derived: If acute stress experience invariably triggers a default compensatory mechanism of tonically increased task shielding, the dual-task context would predict less between-task interference (crosstalk) for stressed individuals than for controls. In contrast, if acute stress experience results in a compensatory mechanism that is associated with the most parsimonious demands on the available resources, the dual-task context would predict the adoption of a resource-saving task-processing mode of reduced task shielding by stressed individuals, leading to increased between-task interference for stressed participants as compared to controls.

In addition to the main focus of testing these opposing predictions, we aimed to address two further questions. First, because previous findings regarding acute stress effects on cognitive control have often implied increased interindividual variance in the stress group as compared to the control group (e.g., Plessow et al., 2012; Qin et al., 2009), we additionally tested for individual-related modulators of stress effects on cognitive performance by applying the action-state orientation concept (e.g., Kuhl, 2000; Kuhl \& Beckmann, 1994). According to this theory, the ability to detach from an unpleasant past experience (captured in the preoccupation dimension) is assumed to especially represent a trait with two manifestations - that is, action-oriented individuals will successfully omit recurring thoughts about unpleasant past events, and state-oriented individuals will get absorbed by them. Therefore, we aimed to determine whether participants with different markedness on the preoccupation scale differ in the extents to which acute psychosocial stress may affect their task shielding during dual-task performance. Finally, since the effects of acute psychosocial stress on task shielding during single-task conductance were found to be time-dependent (i.e., they only developed with increasing time lag to the stressor; Plessow et al., 2011), we measured dual-task performance in two parts, covering two different time intervals with regard to stress exposure and the organism's response to it, in order to examine the time axis of potential stress-induced modulations of task shielding during dual-task performance (for a similar proceeding, see Plessow et al., 2012).

To test the outlined predictions with respect to acute stress and task shielding in dual-task performance, we administered acute stress by applying a well-established psychosocial stress-induction protocol (i.e., the Trier Social Stress Test [TSST]; Kirschbaum, Pirke, \& Hellhammer, 1993). The success of the stress induction was validated using biological and subjective stress markers (i.e., salivary $\alpha$-amylase [sAA] and salivary cortisol, as markers of SNS and HPA-axis activity, respectively—see, e.g., Kirschbaum \& Hellhammer, 1994; Nater \& Rohleder, 2009; participants also filled out the German MDBF mood questionnaire to assess their current mental state; Steyer, Schwenkmezger, Notz, \& Eid, 1997). To target cognitive control processes of task shielding in dual-task performance, the participants were instructed to perform two choice-reaction tasks in close temporal succession. To ensure maximum between-task interference (crosstalk), we used tasks with identical S-R rules (e.g., Fischer, Miller, \& Schubert, 2007; Logan \& Schulkind, 2000). More precisely, the participants were to categorize single-digit numbers as odd or even, first in $\mathrm{T} 1$ and then in T2. As a typical finding, categorization of the target stimulus for Task 1 (S1) is often affected by the simultaneous categorization of the target stimulus for Task 2 (S2), denoting the response-category match effect (e.g., Fischer et al., 2007a; Hommel, 1998; Logan \& Schulkind, 2000; Miller, 2006; Schubert, Fischer, \& Stelzel, 2008; Schuch \& Koch, 2004; see Lien \& Proctor, 2002, for an overview), which is especially detrimental when $\mathrm{S} 2$ calls for a different categorization than $\mathrm{S} 1$ does (e.g., $\mathrm{S} 1=$ odd and $\mathrm{S} 2=$ even). Therefore, the response-category match effect designates the amount of between-task interference, and thus the amount of task shielding (Fischer \& Hommel, 2012). To reiterate, strong task shielding is associated with small 
response-category match effects and would be expected if increases in task shielding represent a default compensatory mechanism during acute stress. Conversely, weak task shielding is associated with large response-category match effects and would be expected if acute stress leads to task performance with priority to sparing the available resources.

\section{Methods}

\section{Participants}

Fifty-six volunteers (28 men, 18-30 years of age, mean age $\pm S D=22.63 \pm 2.79$ years) took part in the study. All of them were healthy, non-medicated, and of normal weight (as indexed by the body-mass index [BMI], $17<\mathrm{BMI}<28$; mean $\mathrm{BMI} \pm S D=22.40 \pm 2.50$ ), and all had normal or corrected-to-normal vision. Fifty-three of the participants claimed right-handedness. None of the participants reported any acute or chronic stress condition. Due to the findings that both habitual smoking and oral contraceptive intake attenuate the physiological stress response, in terms of a smaller stress-related increase in free salivary cortisol (Kirschbaum, Kudielka, Gaab, Schommer, \& Hellhammer, 1999; Rohleder \& Kirschbaum, 2006), we tested only nonsmokers and female volunteers who did not use hormone-based birth control. All of the participants gave their written informed consent prior to inclusion in the study, in accordance with the 1964 Declaration of Helsinki, and received financial compensation or course credit.

\section{Stress induction and stress validation}

The participants were randomly assigned to two experimental groups, with 14 men and 14 women in each group. The stress group was exposed to the TSST protocol (Kirschbaum et al., 1993). During this standardized stress-induction procedure, participants first received a verbal instruction about the upcoming tasks, followed by a 5-min anticipatory period. During the subsequent interaction phase, they had to deliver a public speech and perform a mental arithmetic task in front of a committee (for durations of $5 \mathrm{~min}$ each; total TSST time $=15 \mathrm{~min}+5 \mathrm{~min}$ for taking the participant to the treatment room and instruction). The control group, however, underwent a standardized control situation that widely resembled the TSST protocol but omitted its stress-inducing features (for details, see Het, Rohleder, Schoofs, Kirschbaum, \& Wolf, 2009).

Over the course of the session, at eight time points (i.e., 15,5 , and $1 \mathrm{~min}$ before and $1,10,20,30$, and $40 \mathrm{~min}$ following treatment), saliva samples were collected using Salivette sampling devices (Sarstedt, Nümbrecht, Germany). Via a quantitative enzyme-kinetic method, sAA activity was obtained (cf. Rohleder \& Nater, 2009). Applying a chemiluminescence immunoassay (IBL International, Hamburg, Germany), free-cortisol levels were estimated. In order to additionally assess subjective stress experience, the MDBF mood questionnaire (Steyer et al., 1997) was employed, which displays the current mental state of an individual on three dimensions (i.e., good mood vs. bad mood, calmness vs. restlessness, and alertness vs. fatigue). This self-report measure was implemented five times (i.e., at 15 and $1 \mathrm{~min}$ prior to and 1,20, and 40 min after treatment) simultaneously with the saliva sampling, with the solitary exception of measurement time point $-1 \mathrm{~min}$. At this point, saliva sampling took place prior to the instruction for the upcoming treatment, whereas the MDBF was to be answered only immediately after the treatment introduction. As a consequence, the participants gave account of their current mental state while already anticipating the upcoming treatment situation (i.e., TSST or standardized control situation).

\section{Dual task}

In the present study, we implemented a dual-task paradigm that was closely modeled after the study by Logan and Schulkind (2000) and that has proven a reliable tool for the investigation of task shielding in dual-task performance (e.g., Fischer \& Hommel, 2012; Fischer et al., 2007a; Oriet, Tombu, \& Jolicœur, 2005). In this paradigm, participants performed the same single-digit categorization in Tasks 1 and 2 to maximize the feature overlap between tasks. The measure of interest was Task 1 performance (i.e., error rates and response times [RTs]).

Two target stimuli (i.e., the digits 1-9, except for 5), with a size of $2-3 \mathrm{~mm}$ horizontally and $5 \mathrm{~mm}$ vertically, were presented one above the other in white against black on a 17-in. monitor of an IBM-compatible personal computer. The upper digit denoted the target stimulus for Task 1 (S1), whereas the lower digit represented the target stimulus for Task 2 (S2). The participants were instructed to categorize both stimuli with regard to their parity by providing manual responses on a standard QWERTZ keyboard. For Task 1, the participants responded with the right index finger ("AltGr" key) and the middle finger ("." key) to odd and even targets, respectively. For Task 2, homologously, odd targets had to be answered with the left index finger ("Alt" key), whereas even targets required a left middle finger response ("Y" key).

Each trial started with the presentation of a fixation display consisting of four horizontal lines $(2.5 \mathrm{~mm}$ in length, two $4.5 \mathrm{~mm}$ above and two $4.5 \mathrm{~mm}$ below screen center, the upper and lower lines each displaced $5 \mathrm{~mm}$ to the left and right of a notional line through screen center) indicating the space between a left and a right line on the upper and the lower parts of the display as presentation locations for the 
upcoming S1 and S2, respectively (total fixation display size: $12 \times 14 \mathrm{~mm}$, resulting in a visual angle of $1.15^{\circ} \times 1.34^{\circ}$ at a viewing distance of $60 \mathrm{~cm}$ ). After $500 \mathrm{~ms}$, S1 was displayed at the upper target-stimulus location. With a stimulus-onset asynchrony (SOA) of 50,150, or $900 \mathrm{~ms}$, S2 was added at the lower target-stimulus location. The participants were instructed to first respond as quickly and accurately as possible to $\mathrm{S} 1$, and only subsequently to respond also as quickly and accurately as possible to S2 (Task 1 priority instruction). Furthermore, the instructions stressed that participants refrain from delaying their response to S1. The delayed S2 onset facilitated the prioritization of Task 1 processing (in addition to the instructions) and allowed for a systematic manipulation of the overlap (i.e., the task load) of dual-task processing. Both stimuli were displayed for $1,000 \mathrm{~ms}$ (plus SOA, for S1), followed by $800 \mathrm{~ms}$ of blank screen, providing a total response window of 1,800 ms from S2 onset. Within all trials, feedback was provided for $300 \mathrm{~ms}$. If both answers were given correctly, the word "richtig" (correct) was displayed. If at least one response was erroneous, "falsch" (false) was reported. If less than two answers were given within the response window, "zu langsam" (too slow) was presented. Following a random blank-screen interval between 100 and 1,000 ms, the next trial started. The stimulus presentation and data recording were implemented using Presentation software (Version 0.71; Neurobehavioral Systems, Inc., Albany, CA, USA).

\section{Action-state orientation}

To assess the disposition toward action-state orientation in preoccupation situations, all participants completed the action-control scale (ACS-90; Kuhl, 1994). According to the total score on the preoccupation subscale, the ACS allowed us to differentiate individuals with a disposition toward state orientation $(<5)$ from individuals who primarily orient themselves toward their current action $(\geq 5)$ following on an unpleasant experience. This resulted in 12 state-oriented and 16 action-oriented individuals within the stress group and 11 state-oriented and 17 action-oriented volunteers in the control group, $\chi^{2}(1, N=56)=0.07, p=.79$.

\section{Procedure}

We first trained the cognitive task (total number of training trials $=132)$ to familiarize participants with the dual-task procedure, and thereby to minimize practice and learning effects during posttreatment cognitive testing. At $30 \mathrm{~min}$ after arrival, the participants underwent the treatment (i.e., TSST or standardized control situation). At $5 \mathrm{~min}$ after treatment completion, the first part of cognitive testing started (five blocks comprising 48 trials each $=240$ trials). The second part of the dual task followed with a time lag of $25 \mathrm{~min}$ from the end of treatment (five blocks of 48 trials each $=240$ trials; total number of test trials $=480$, total session duration $=100 \mathrm{~min}$ ).

Sessions were scheduled between 11:30 a.m. and 8 p.m. Due to evidence for an influence of instantaneous food and caffeine intake on the cortisol stress response assessed via saliva (Gonzalez-Bono, Rohleder, Hellhammer, Salvador, \& Kirschbaum, 2002; Lovallo, Farag, Vincent, Thomas, \& Wilson, 2006), participants were asked to refrain from eating and from consuming sugary or caffeinated beverages $2 \mathrm{~h}$ prior to their appointed time. Since acute glucose availability is assumed to be essential for a stress-related HPA-axis activity increase (Kirschbaum et al., 1997), all participants received $200 \mathrm{ml}$ of grape juice at the beginning of the session to elevate their blood glucose levels and to allow these to converge across individuals.

\section{Data analysis}

In order to address changes in individual physiological and subjective stress levels over the course of the session, repeated measures analyses of variance (ANOVAs), comprising the within-subjects factor Measurement Time Point (eight or five levels, respectively) and the between-subjects factor Stress (stress vs. no stress) were conducted on sAA, cortisol, and the total scores on the three MDBF dimensions. In preparation for the statistical analyses (i.e., to ensure compliance with the requirements of general-linear-model-based procedures in terms of Gaussian distribution), the sAA and cortisol data were first logarithmized to base 10. Dual-task performance was analyzed by entering the within-subjects factors Response-Category Match (mismatch vs. match), SOA (50 vs. 150 vs. $900 \mathrm{~ms}$ ), and Part (first vs. second), as well as the between-subjects factors Stress (stress vs. no stress) and Action-State Orientation (action-oriented vs. state-oriented) into repeated measures ANOVAs on the error rates and mean RTs in Task 1 . To eliminate the possibility of perceptual matching, trials with identical target stimuli in both tasks $(12.65 \%)$ were excluded. For the RT analysis only, error trials $(9.03 \%)$ and RTs differing by more than 2.5 standard deviations from the means of the individual-cell mean RTs in both tasks (3.37\%) were also omitted. Greenhouse-Geisser corrections were applied where appropriate.

\section{Results}

Stress response

Neuroendocrine measures Stressed individuals and controls differed in their cortisol time courses, $F(7,378)=32.42, p<$ $.001, \eta_{\mathrm{p}}{ }^{2}=.38$. While no group differences occurred prior to TSST and the standardized control situation, respectively, all $p \mathrm{~s} \geq .29$, following treatment the stress group constantly 
displayed higher cortisol levels than did the control group: 1 min, $t(54)=2.31, p<.05 ; 10 \min , t(54)=4.67, p<.001$; $20 \mathrm{~min}, t(54)=4.91, p<.001 ; 30 \mathrm{~min}, t(54)=4.28, p<.001$; and $40 \mathrm{~min}, t(54)=3.70, p<.01$. For sAA, the Measurement Time Point $\times$ Stress interaction did not reach significance, $F(7$, $378)=1.88, p=.11, \eta_{\mathrm{p}}{ }^{2}=.03$. Post-hoc comparisons for the single measurement time points (parallel to the cortisol-level analysis) revealed that, relative to controls, stressed individuals showed higher sAA activity solely immediately after treatment $(1 \mathrm{~min}), t(54)=2.42, p<.05$, all other $p \mathrm{~s} \geq .14$ (Fig. 1 top).

Mental state The MDBF data analyses revealed different time courses for the stress group and the control group with respect to the two dimensions of good mood versus bad mood and calmness versus restlessness, $F(4,216)=$
3.62, $p<.05, \eta_{\mathrm{p}}{ }^{2}=.06$, and $F(4,216)=4.68, p<.01$, $\eta_{\mathrm{p}}{ }^{2}=.08$. More precisely, stressed participants displayed worse mood directly after treatment $(1 \mathrm{~min})$, relative to controls, $t(54)=2.73, p<.01$, and a trend toward worse mood after receiving the treatment instructions but prior to the actual treatment $(-1 \mathrm{~min}), t(54)=1.73, p=.09$ (all other $p s \geq .20)$. Immediately following treatment, the volunteers in the stress group also reported more restlessness than did controls, $t(54)=2.62, p<.05$, whereas no group differences regarding calmness versus restlessness were found for all other measurement time points, $p \mathrm{~s} \geq .14$. Fatigue increased throughout the session, $F(4$, 216) $=26.29, p<.001, \eta_{\mathrm{p}}{ }^{2}=.33$, developing equally in both the stress and control group, $F(4,216)=1.13$, $p=.33, \eta_{\mathrm{p}}{ }^{2}=.02$. The mean fatigue levels, too, were similar in both groups, $F<1$ (Fig. 2).
Fig. 1 (Top) Mean levels of salivary $\alpha$-amylase (sAA) and salivary cortisol as a function of time (minutes before or after treatment) for the stress group and the control group. (Bottom) Error rates in Task 1 as a function of response-category match (mismatch vs. match) for the stress group and the control group and for Dual-Task Parts 1 and 2. Error bars represent standard errors of the means. ${ }^{*} p<.05 .{ }^{* *} p<.01{ }^{* * * *} p<.001$

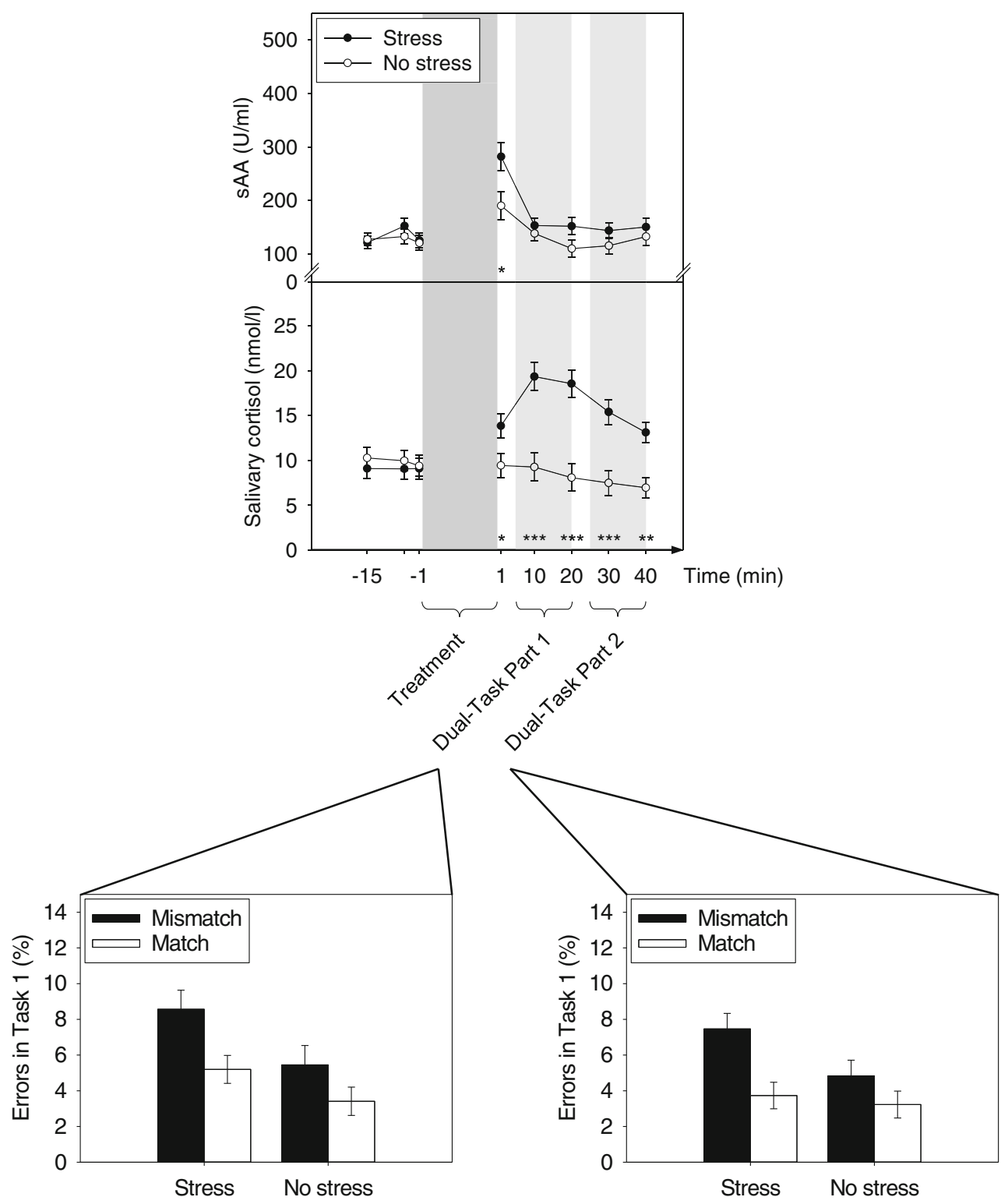




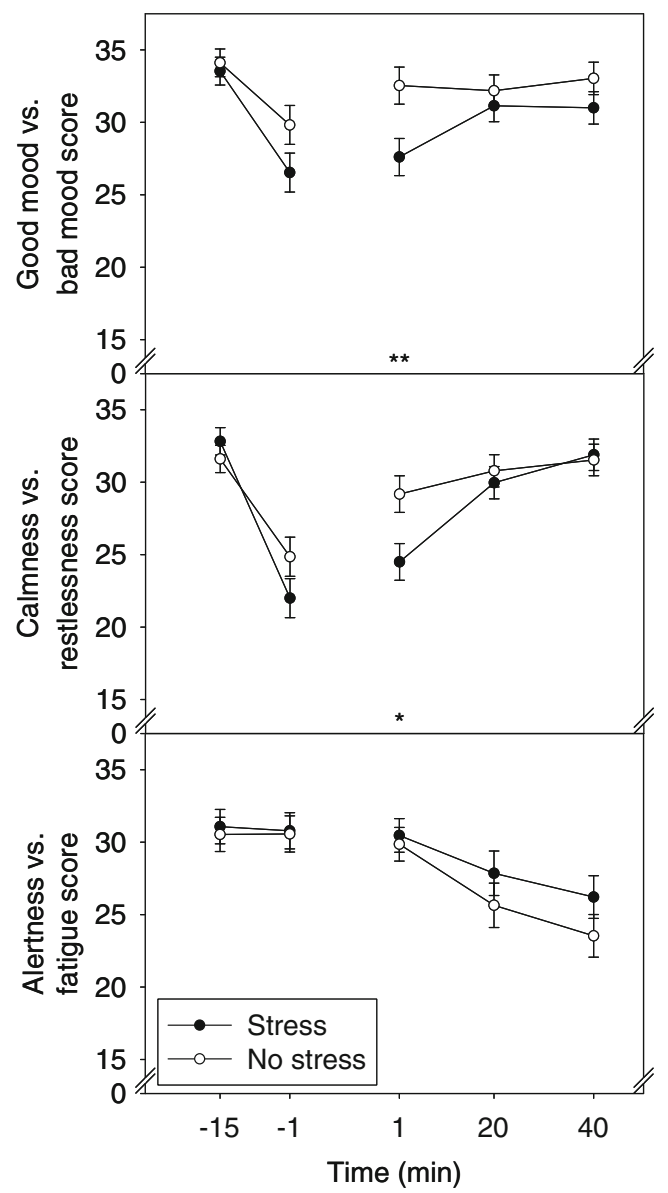

Fig. 2 Mental state measured on the three dimensions good mood versus bad mood, calmness versus restlessness, and alertness versus fatigue, assessed by the MDBF mood questionnaire (Steyer et al., 1997), as a function of time (minutes before or after treatment) for the stress group and the control group. The mental-state measurement time point -1 min took place directly after the instruction for the subsequent treatment (i.e., Trier Social Stress Test or standardized control situation), within the anticipatory period. Error bars represent standard errors of the means. ${ }^{*} p<.05 .{ }^{*} p<.01$

\section{Cognitive performance}

The results of Task 1 performance (error rates and RTs) are presented in Table 1 and Fig. 1 (bottom). ${ }^{3}$

\section{Error rates}

Dual-task performance Significant main effects were found for both response-category match (mismatch, $6.58 \%$; match, $3.89 \%), F(1,52)=49.40, p<.001, \eta_{\mathrm{p}}{ }^{2}=.49$, and SOA (50 ms, $6.12 \%$; $150 \mathrm{~ms}, 5.46 \%$; $900 \mathrm{~ms}, 4.05 \%$ ), $F(2,104)=10.06, p<.001, \eta_{\mathrm{p}}^{2}=.16$. Furthermore, the effect of response-category match decreased with increasing SOA (50 ms, $4.81 \%$; $150 \mathrm{~ms}, 3.41 \%$; $900 \mathrm{~ms},-0.16 \%$ ), $F$ $(2,104)=20.14, p<.001, \eta_{\mathrm{p}}{ }^{2}=.28$.

\footnotetext{
${ }^{3}$ For the Task 2 results, see the supplementary materials.
}

Effects of acute psychosocial stress and action-state orientation on dual-task performance Most important for the present study, the response-category match effect was modulated by stress, $F(1,52)=5.10, p<.05, \eta_{\mathrm{p}}^{2}=.09$. More precisely, stressed participants displayed a larger response-category match effect $(3.55 \%)$ than did controls $(1.82 \%)$. Post-hoc $t$ tests with independent measures showed that this increased response-category match effect primarily originated from stress-related error rate increases in response-category mismatch trials (stress, $8.02 \%$; no stress, $5.14 \%), t(54)=2.01, p<.05$ (one-tailed). In contrast, no group difference was found for response-category match trials, $t(54)=1.02, p=.31$. In order to test whether the observed effect of stress on the response-category match effect on the group-comparison level was also evident on the individual level, we correlated the total increase in neuroendocrine measures following treatment ${ }^{4}$ with the amount of between-task interference (i.e., the responsecategory match effect) of each individual. This led to a significant correlation between the cumulative increase in salivary cortisol following treatment and the responsecategory match effect, $r \mathrm{~s}(56)=.32, p<.05$. The larger the total increase in salivary cortisol, the more pronounced the response-category match effect. In contrast, treatment-related changes in SAA did not correlate with the response-category match effect, $r \mathrm{~s}(56)=-.07, p=.62$.

A larger response-category match effect was also found for state-oriented individuals (3.58\%) than for action-oriented participants (1.79\%; Fig. 3), which is reflected in the interaction between Response-Category Match and action-state orientation, $F(1,52)=5.49, p<.05, \eta_{\mathrm{p}}{ }^{2}=.10$. Importantly, the effects of stress and action-state orientation on the responsecategory match effect did not interact, $F(1,52)=1.54, p=.22$, $\eta_{\mathrm{p}}{ }^{2}=.03$. The aforementioned decrease of error rates with increasing SOA seemed slightly pronounced in the stress group (50 ms, $7.63 \%$; $150 \mathrm{~ms}, 6.70 \%$; $900 \mathrm{~ms}, 4.40 \%$ ) as compared to the control group $(50 \mathrm{~ms}, 4.77 \% ; 150 \mathrm{~ms}$, $4.23 \%$; $900 \mathrm{~ms}, 3.70 \%$ ). This observation, however, fell just short of significance, $F(2,104)=2.78, p=.07, \eta_{\mathrm{p}}{ }^{2}=.05$. At the same time, the decrease of the response-category match effect with increasing SOA was unaffected by stress, $F(2,104)=1.56, p=.22, \eta_{\mathrm{p}}{ }^{2}=.03$. Also, neither the SOA effect nor the Response-Category Match $\times$ SOA interaction was affected by action-state orientation, either alone or combined with stress, $p \mathrm{~s} \geq .15$. Overall, the mean error rates were numerically larger in stressed individuals $(6.24 \%)$ than in controls $(4.23 \%)$. This difference, however, only approached

\footnotetext{
${ }^{4}$ Calculated as the area under the curve with respect to increase (Prüssner, Kirschbaum, Meinlschmid, \& Hellhammer, 2003) in logarithmized data from the measurement time point immediately prior to treatment (i.e., $-1 \mathrm{~min}$, baseline), as well as from all measurement time points subsequent to treatment (i.e., 1, 10, 20, 30, and $40 \mathrm{~min}$ after treatment cessation).
} 
Table 1 Error rates (\%) and response times (RTs) in Task 1 for all combinations of response-category match and stimulus-onset asynchrony (SOA) for the stress group and the control group overall, as well as separately for Dual-Task Parts 1 and 2

\begin{tabular}{|c|c|c|c|c|c|c|c|}
\hline \multirow{2}{*}{$\begin{array}{l}\text { Response-Category } \\
\text { Match }\end{array}$} & \multirow[t]{2}{*}{ SOA (ms) } & \multicolumn{3}{|c|}{ Stress $(n=28)$} & \multicolumn{3}{|c|}{ No Stress $(n=28)$} \\
\hline & & Overall & Dual-Task Part 1 & Dual-Task Part 2 & Overall & Dual-Task Part 1 & Dual-Task Part 2 \\
\hline \multicolumn{8}{|l|}{ Errors } \\
\hline \multirow[t]{3}{*}{ Mismatch } & 50 & $10.31(1.31)$ & $11.93(1.71)$ & $8.70(1.20)$ & $6.90(1.32)$ & $7.16(1.73)$ & $6.63(1.22)$ \\
\hline & 150 & $9.25(1.19)$ & $8.88(1.23)$ & $9.61(1.35)$ & $5.09(1.20)$ & $5.43(1.24)$ & $4.75(1.37)$ \\
\hline & 900 & $4.49(0.68)$ & $4.90(0.85)$ & $4.09(0.73)$ & $3.44(0.68)$ & $3.76(0.86)$ & $3.12(0.74)$ \\
\hline \multirow[t]{3}{*}{ Match } & 50 & $4.94(0.81)$ & $5.16(0.98)$ & $4.72(0.85)$ & $2.65(0.82)$ & $2.64(1.00)$ & $2.66(0.87)$ \\
\hline & 150 & $4.15(0.93)$ & $4.97(1.05)$ & $3.34(1.00)$ & $3.36(0.94)$ & $3.84(1.07)$ & $2.88(1.02)$ \\
\hline & 900 & $4.30(0.74)$ & $5.46(0.95)$ & $3.14(0.87)$ & $3.95(0.75)$ & $3.76(0.96)$ & $4.15(0.88)$ \\
\hline \multicolumn{8}{|l|}{ RTs } \\
\hline \multirow[t]{3}{*}{ Mismatch } & 50 & 824 (24) & $818(24)$ & $829(25)$ & $780(24)$ & $772(24)$ & $787(25)$ \\
\hline & 150 & $805(22)$ & $809(21)$ & $801(25)$ & $753(23)$ & $742(22)$ & $765(25)$ \\
\hline & 900 & $802(51)$ & $810(51)$ & $794(53)$ & $682(52)$ & $656(52)$ & $708(54)$ \\
\hline \multirow[t]{3}{*}{ Match } & 50 & 724 (17) & $726(17)$ & $723(18)$ & $691(17)$ & $692(17)$ & $690(18)$ \\
\hline & 150 & $742(18)$ & 751 (19) & $732(18)$ & $701(18)$ & 701 (19) & $701(18)$ \\
\hline & 900 & $791(50)$ & $794(50)$ & $788(52)$ & $684(51)$ & $664(51)$ & $705(53)$ \\
\hline
\end{tabular}

$N=56$. Values are given as means with standard errors of the means (in parentheses).

significance, $F(1,52)=3.29, p=.08, \eta_{\mathrm{p}}{ }^{2}=.06$. No main effect of action-state orientation on error rates was revealed, $F(1,52)=2.42, p=.13, \eta_{\mathrm{p}}^{2}=.04$. Furthermore, stress and action-state orientation did not interact, $F(1,52)=1.21$, $p=.28, \eta_{\mathrm{p}}^{2}=.02$.

To test whether the observed effects depended on gender, we repeated the original ANOVA with all a priori factors and the additional between-subjects factor Gender. The results showed no modulation by gender of the revealed effects of stress and action-state orientation on the response-category match effect, both $F_{\mathrm{s}}<1$. Moreover, gender interacted neither with stress alone nor with any factorial combination including stress, all $p \mathrm{~s} \geq .16$.

Comparison of the two parts Overall, the mean error rate decreased from Dual-Task Part 1 (5.66 \%) to Dual-Task Part

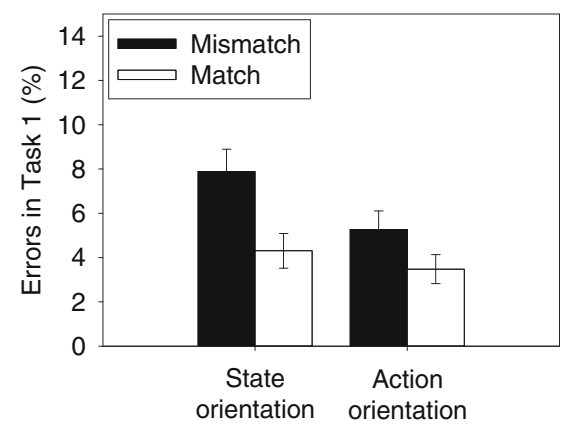

Fig. 3 Error rates in Task 1 as a function of response-category match (mismatch vs. match) for state-oriented and action-oriented individuals. Error bars represent standard errors of the means
$2(4.82 \%), F(1,52)=5.85, p<.05, \eta_{\mathrm{p}}{ }^{2}=.10$. In contrast, the effects of both response-category match and SOA remained unchanged, $F \mathrm{~s}<1$, as did the reduction of the response-category match effect with increasing SOA, $F(2,104)=1.79, p=.17, \eta_{\mathrm{p}}{ }^{2}=.03$. The modulating influences of stress and action-state orientation on the response-category match effect did not differ between the dual-task parts, both $F \mathrm{~s}<1$. No further interactions of dual-task part with one or more of the other factors reached significance, all $p \mathrm{~s} \geq 09$.

Yet, in order to draw clear conclusions regarding the occurrence intervals of a reliable stress impact on the response-category match effect (Plessow et al., 2011), it seems inevitable that we should additionally demonstrate that the stress impact on the response-category match effect can be substantiated by the appropriate inference statistics when separately analyzing Dual-Task Parts 1 and 2 (see also Fig. 1). Following this reasoning, we repeated the original ANOVA (excluding the within-subjects factor Part) for both cognitive-testing parts separately. For the first part of the dual task, the response-category match effect was only numerically larger for the stress group $(3.37 \%)$ than for the control group $(2.04 \%)$, but it was not statistically reliable, $F(1,52)=2.06, p=.16, \eta_{\mathrm{p}}{ }^{2}=.04$. The main effect of stress on error rates, with larger error rates for stressed individuals $(6.88 \%)$ than for controls $(4.43 \%)$ in Dual-Task Part 1 was close to significance, $F(1,52)=3.84, p=.06$, $\eta_{\mathrm{p}}{ }^{2}=.07$. Within the second part of the dual task, the response-category match effect was larger in the stress group $(3.73 \%)$ than in the control group $(1.61 \%), F(1,52)=6.11$, 
$p<.05, \eta_{\mathrm{p}}{ }^{2}=.11$. Equivalently to the stress impact on the overall response-category match effect, this difference between the stress and control group was driven by larger error rates in response-category mismatch trials for stressed participants $(7.47 \%)$ than for controls $(4.84 \%), t(54)=1.92, p<.05$ (one-tailed). Error rates did not differ between the groups in response-category match trials, $t<1$. At the same time, the main effect of stress on error rates was not significant, $F(1,52)=2.16, p=.15, \eta_{\mathrm{p}}{ }^{2}=.04$ (Fig. 1 bottom).

\section{RTs}

Dual-task performance The RT analysis revealed a significant main effect of response-category match (mismatch, $774 \mathrm{~ms}$; match, $722 \mathrm{~ms}), F(1,52)=114.29, p<.001, \eta_{\mathrm{p}}{ }^{2}=$ .69. Moreover, the response-category match effect decreased with increasing SOA (50 ms, $94 \mathrm{~ms} ; 150 \mathrm{~ms}, 58 \mathrm{~ms} ; 900 \mathrm{~ms}$, $4 \mathrm{~ms}), F(2,104)=46.54, p<.001, \eta_{\mathrm{p}}{ }^{2}=.47$. SOA alone did not affect RTs, $F<1$.

Effects of acute psychosocial stress and action-state orientation on dual-task performance There was only a trend toward larger overall mean RTs in stressed participants (781 ms) than in controls $(715 \mathrm{~ms}), F(1,52)=2.77, p=.10$, $\eta_{\mathrm{p}}{ }^{2}=.05$. Similarly, the response-category match effect was numerically, but not statistically, larger for the stress group $(58 \mathrm{~ms})$ than for the control group $(46 \mathrm{~ms}), F(1,52)=1.44$, $p=.24, \eta_{\mathrm{p}}{ }^{2}=.03$, and was neither affected by action-state orientation nor by the combination of both factors, $F_{\mathrm{S}}<1$.

Comparison of the two parts RTs were not significantly affected by part, $F(1,52)=1.70, p=.20, \eta_{\mathrm{p}}{ }^{2}=.03$. However, a significant interaction between part and stress was found, $F(1,52)=6.10, p<.05, \eta_{\mathrm{p}}{ }^{2}=.11$. Whereas the stress group did not differ between Dual-Task Parts 1 and 2 (788 and $780 \mathrm{~ms}$, respectively), $t(27)=1.02, p=.32$, the RTs of the control group were slower in Dual-Task Part 2 $(731 \mathrm{~ms})$ than in Dual-Task Part $1(709 \mathrm{~ms}), t(27)=2.70$, $p<.05$. This effect seemed especially pronounced at the long SOA, resulting in the close-to-significant interaction SOA $\times$ Part $\times$ Stress, $F(2,104)=3.31, p=.06, \eta_{\mathrm{p}}{ }^{2}=.06$ (see also Table 1). Regarding action-state orientation, stateoriented individuals displayed a trend toward larger RTs in the second $(761 \mathrm{~ms})$ than in the first part of the dual task (744 ms), while numerical inspection revealed no such difference for action-oriented individuals (Part 1, $746 \mathrm{~ms}$; Part 2, $743 \mathrm{~ms}), F(1,52)=3.01, p=.09, \eta_{\mathrm{p}}{ }^{2}=.06$. Stateoriented individuals showed an increase in RTs from the first to the second cognitive-testing part primarily for the long SOA, which is reflected in a significant three-way interaction between SOA, part, and action-state orientation, $F(2,104)=$ $3.82, p<.05, \eta_{\mathrm{p}}{ }^{2}=.07$. Importantly, the four-way interaction SOA $\times$ Part $\times$ Stress $\times$ Action-State Orientation was not significant, $F(2,104)=2.26, p=.13, \eta_{\mathrm{p}}{ }^{2}=.04$. Independent of the between-subjects factors, there was a trend toward a larger response-category match effect in Dual-Task Part 2 $(58 \mathrm{~ms})$ as compared to Part $1(47 \mathrm{~ms}), F(1,52)=3.68$, $p=.06, \eta_{\mathrm{p}}{ }^{2}=.07$. No further significant interactions occurred, all $p \mathrm{~s} \geq .20$.

General quality of dual-task performance

The quality of dual-task performance is often reported in total performance measures such as the added RTs of both tasks (e.g., Miller et al., 2009). In the present study, we did not find significant effects in total error rates (stress, $9.72 \%$; no stress, $8.34 \%$ ), $t<1$, or total RTs (stress, $1,571 \mathrm{~ms}$; no stress, $1,468 \mathrm{~ms}), t(54)=1.59, p=.12$.

\section{Discussion}

The present study investigated the impact of acute psychosocial stress on cognitive control processes of task shielding in a dual-task context. Following a previous single-task study that showed tonically increased levels of task shielding under acute psychosocial stress (Plessow et al., 2011), we aimed at testing whether these tonically increased levels of task shielding represent a default compensatory mechanism that is reflexively triggered by acute stress experience or whether acute stress results in adopting the most parsimonious task-processing mode in order to spare mental resources.

These assumptions are testable in dual-task performance, in which a task-processing mode with increased levels of task shielding is also concomitant with increased mental effort and resource load (Lehle et al., 2009). Therefore, in a dual-task context, a default compensatory mechanism of increased task shielding would reduce between-task interference (i.e., lead to a smaller response-category match effect) under acute stress and should be observable even when this mechanism does not reflect the resource-conserving taskprocessing mode. If the response to an acute stress experience, however, relies on the adoption of a resource-conserving taskprocessing mode, within a dual-task situation, task shielding should be less strong under conditions of acute stress, resulting in increased between-task interference (i.e., a larger response-category match effect) for stressed individuals than for controls.

The results are straightforward: First, as expected, the applied stress-induction protocol (i.e., TSST) reliably evoked the typical two-component physiological stress response, comprising an increase in both SNS and HPA-axis activity, as indicated by increased sAA activity and elevated salivary cortisol levels in the stress group as compared to the control group at different time points following treatment. Second, the stress group displayed a larger response- 
category match effect in Task 1, and thus less task shielding than the control group. On an individual level, this was expressed by a significant correlation between the individual treatment-related changes in cortisol levels as a marker for HPA-axis activity and the response-category match effect (see below for a more detailed discussion).

These findings of reduced task shielding in dual-task performance under conditions of acute stress contradict the assumption of a unitary consequence of acute stress on cognitive control processes in terms of increased task shielding. This hypothesis can be traced to early theoretical work suggesting that stress narrows attention and internal processing (Easterbrook, 1959), and thus predicting increased rather than reduced task shielding. Easterbrook's idea of stressinduced attentional focusing has received recent empirical support-for example, in demonstrations of reduced interference from competing automatic response tendencies when performing a Stroop task under potentially stressful conditions (e.g., Chajut \& Algom, 2003; Huguet, Galvaing, Monteil, \& Dumas, 1999), as well as in our own previous work, in which we showed that an acute stress experience results in tonically increased task shielding at the cost of reduced flexibility in a selective-attention task (Plessow et al., 2011).

The apparently conflicting findings of the present study and the assumption of increased task shielding as a compensatory mechanism under stress (in line with Easterbrook's narrowed-attention account) can be reconciled with our alternative hypothesis, in which we assume that acute stress triggers the adoption of a task-processing mode that is less resource-demanding. As mentioned earlier, in a single-task situation, a tonic increase in task shielding seems to represent a less hazardous and resource-demanding taskprocessing mode that is favored over the usual flexible trialto-trial adaptation of task shielding to response-conflict occurrence under acute stress (Plessow et al., 2011). In a dual-task context, however, the less resource-consuming task-processing mode is associated with reduced task shielding that increases parallel processing (Lehle et al., 2009) for example, by relaxing between-task inhibition (Koch et al., 2010). ${ }^{5}$

\footnotetext{
$\overline{{ }^{5} \text { The view of }}$ relaxed task shielding under stress receives further support from the Task 2 performance (see the supplementary materials). In Task 2, the response-category match effect reversed at the long SOA, which might be interpreted as a rebound effect of strong between-task inhibition. More precisely, increased Task 1 shielding might result in repetition costs at the long SOA when the same categorization is repeated in Task 2 (i.e., positive priming becomes interference). Interpreting this reversal as an inhibitory rebound effect, it is interesting to note that this rebound was reduced for the stress group as compared to the control group, which can be taken as evidence for a relaxation of between-task inhibition under stress (we thank Iring Koch for highlighting this possibility).
}

The adoption of a task-processing mode that saves available resources under stress is in line with ideas highlighting the relevance of additional stress-coping mechanisms occupying resources that are consequently not available for other resource-dependent cognitive processes (e.g., Kahneman, 1973; Keinan, Friedland, Kahneman, \& Roth, 1999). In these terms, we may conclude that stressed individuals adopted a resource-saving mode of dual-task processing. Thereby, the question of whether limited resources coercively necessitated the resource-sparing task-performance mode or whether its adoption was the result of resource reallocation processes triggered by acute stress experience (e.g., as proposed in the cognitive-energetical framework of Hockey, 1997), and thus of a "choice" made between two possible task-processing modes, remains to be answered.

Arnsten (2009) has gone even further by concluding that "acute uncontrollable stress impairs PFC-mediated cognitive functions in humans and animals and switches the control of behaviour and emotion to more primitive brain circuits" (Arnsten, 2009, p. 412). On a related note, Schwabe, Wolf, and Oitzl (2010) stated that stress experience leads to a stronger recourse to neostriatum-dependent rigid "habit" memory and a reduction in the use of hippocampus- and PFC-dependent flexible "cognitive" memory.

Supporting the assumption of impaired PFC-dependent cognitive functions under stress, studies have revealed impairments in suppressing ongoing responses (Scholz et al., 2009), in the flexible implementation of task goals (e.g., Liston et al., 2009; Plessow et al., 2012), and in working memory processes serving to maintain task-relevant information (e.g., Elzinga \& Roelofs, 2005; Oei et al., 2006). On a more general level, stress was found to reduce cognitive flexibility during creative-thinking and problem-solving tasks (Alexander, Hillier, Smith, Tivarus, \& Beversdorf, 2007), as well as the use of negative feedback (Petzold, Plessow, Goschke, \& Kirschbaum, 2010).

In our view, the results of the present study do not support the assumption of a global impairment in PFC-mediated cognitive functions. Even though stressed participants were exposed to a stress-induction method (TSST) that is perceived as ego-threatening and uncontrollable (cf. Dickerson \& Kemeny, 2004), they were, nevertheless, able to maintain overall high dual-task performance levels (as indicated, for example, by a total performance level that did not significantly differ from that of the controls). Therefore, in the context of dual tasks, we conclude that acute stress induces a shift in the recruitment of different cognitive control processes and task-processing modes (see also Plessow et al., 2011) rather than a shift away from the involvement of cognitive control. Thereby, choosing the less resource-demanding task-processing mode may also result in action control on the basis of mostly automatized S-R links (e.g., Schwabe et al., 2010). Yet the strong assumption of 
a loss of prefrontal regulation under conditions of acute stress may, at least in the face of successful dual-task performance, not be universally valid.

The assumption that acute stress results in the adoption of a resource-saving task-processing mode (i.e., reduced task shielding) is of high relevance for theories on dual-tasking and seems to support theoretical notions of resource allocation in models of capacity sharing (e.g., Navon \& Miller, 2002; Tombu \& Jolicœur, 2003). According to the task demands and the situational context, available cognitive resources are flexibly allocated between tasks to ensure the most efficient dualtask performance. A threatening acute stress experience seems to draw on resources that are also required by the cognitive control mechanisms enabling efficient prioritized task shielding. Hence, the adaptation of a task-processing mode that is economical in its requirements of cognitive resources can be interpreted as a flexible adjustment of strategic dual-task processing according to parameters of the given situation (Logan \& Gordon, 2001; Meyer \& Kieras, 1997). In these terms, the finding that the amount of task shielding, and thus the shift to a more parallel task-processing mode (cf. Lehle \& Hübner, 2009), is adaptively adjusted according to situational context parameters highlights the role of control processes in dual-task coordination and is difficult to reconcile with traditional postulations of a structural and immutable bottleneck (e.g., Pashler, 1994).

As noted above, the observation of a stress-related decrease in task shielding on the group level was further substantiated on the individual level by a significant correlation between the total increase in salivary cortisol after treatment and the response-category match effect. Thereby, a more pronounced response-category match effect indicating less task shielding was found to be associated with larger increases in salivary cortisol as a marker for HPA-axis activity (but not with treatment-related increases in SAA activity). The assumption of an association between the HPA stress response and the observed stress-related behavioral changes gets further support from the fact that an increased response-category match effect in the stress group relative to the control group was only numerically observed in the first part of the dual task (i.e., 5-20 min after the end of treatment) but was statistically reliable in the second part (i.e., 25-40 min following treatment, during the ongoing HPA stress response but in the absence of stress-related alterations in SNS activity; see also Plessow et al., 2011). Even though this does not preclude the SNS-related stress response from playing a pivotal role in the development of acute stress effects on the investigated cognitive control processes, it strongly highlights the relevance of the HPA stress response for these findings. In addition, at those time points at which the effect occurred, the groups did not differ with regard to their subjective stress experience and/or exertion (e.g., levels of mood, arousal, and fatigue).
A further interesting aspect of the present findings is that stress effects on the cognitive control processes of task shielding were predominantly evident in error rates. Although similar patterns of increased response-category match effects under stress were found numerically for the RTs of T1 and T2, in neither RT did stress significantly affect the response-category match effect. Most importantly, however, our data do not support an interpretation by means of an unspecific performance criterion shift (i.e., speedaccuracy trade-off) in terms of premature responding. It should also be noted that stress effects occurring exclusively in error rates are rather common and have been repeatedly reported by several work groups (e.g., Hsu, Garside, Massey, \& McAllister-Williams, 2003; Plessow et al., 2011; Plessow et al., 2012). Therefore, we believe that this is a systematic effect which is not incidental. Even though a conclusive explanation of this selective influence is still warranted, at least for the present study, we can speculate that one reason why stress effects might be "easier" to obtain in error data could be due to stress effects being especially evident in response-category mismatch trials. Eliminating a larger number of erroneous trials in this condition might have lowered the mean RT level, as these RTs would have potentially been slow trials if the participants had been able to execute the correct response (see also Fischer, Schubert, \& Liepelt, 2007).

To acknowledge potential gender effects in the interaction between acute stress and cognitive functions (e.g., Smeets, Dziobek, \& Wolf, 2009; Wolf, Schommer, Hellhammer, McEwen, \& Kirschbaum, 2001), we purposely recruited $50 \%$ male and $50 \%$ female volunteers who were equally allocated to the stress and control group. Importantly, the observed impact of acute psychosocial stress on processes of cognitive control was not modulated by gender (see also Plessow et al., 2011; Plessow et al., 2012), which suggests stable and universal influences that reveal themselves in a gender-nonspecific manner. Furthermore, in the present study, we accounted for individual dispositions in action-state orientation as a potential modulator of acute psychosocial stress effects on cognitive control parameters by including data from the action-control scale (Kuhl, 1994), on the basis of which individuals are classified as being more action-oriented versus state-oriented, differing with regard to their levels of assumed decisiveness, flexibility in adapting to new situations, and ability to detach from previous unpleasant experiences (cf. Kuhl \& Beckmann, 1994). We found no differences between action-oriented and state-oriented individuals in the extents to which acute psychosocial stress affected their task shielding. Hence, we suppose that stress-related reduced task shielding in dual-task performance is not due to higher levels of preoccupation (e.g., sustained rumination) with the unpleasant stress experience (as would be typical for individuals with a disposition toward state orientation, but not for actionoriented participants). Interestingly, the finding of overall 
increased response-category match effects for state-oriented relative to action-oriented participants, however, might point to either a general predisposition toward decreased taskshielding capability (and/or toward more shared representations of the individual tasks within the dual-task context) in individuals with a disposition toward state orientation.

In summary, with the present study, we have provided evidence that acute psychosocial stress affects the cognitive control processes specifically involved in dual-task performance. More precisely, we demonstrated that acute stress experience results in an adaptation of control settings in a dual-task situation in terms of a shift toward more parallel processing (reduced task shielding). This shows that two pivotal everyday demands-successful performance under stress conditions and simultaneous conductance of multiple tasks - cannot be regarded as independent, but rather interact strongly in determining the performance outcome. Subsequent research will be needed to extend the present research by targeting further cognitive control mechanisms that are also essential for successful dual-task performance (e.g., online order control; Luria \& Meiran, 2003) and to advance our understanding of the underlying biological mediators involved.

\section{References}

Alexander, J. K., Hillier, A., Smith, R. M., Tivarus, M. E., \& Beversdorf, D. Q. (2007). Beta-adrenergic modulation of cognitive flexibility during stress. Journal of Cognitive Neuroscience, 19, 468-478. doi:10.1162/jocn.2007.19.3.468

Arnsten, A. F. (2009). Stress signalling pathways that impair prefrontal cortex structure and function. Nature Reviews Neuroscience, 10, 410-422. doi: $10.1038 / \mathrm{nrn} 2648$

Chajut, E., \& Algom, D. (2003). Selective attention improves under stress: Implications for theories of social cognition. Journal of Personality and Social Psychology, 85, 231-248. doi:10.1037/ 0022-3514.85.2.231

de Kloet, E. R., Joels, M., \& Holsboer, F. (2005). Stress and the brain: From adaptation to disease. Nature Reviews Neuroscience, 6 , 463-475. doi:10.1038/nrn1683

de Kloet, E. R., \& Reul, J. M. (1987). Feedback action and tonic influence of corticosteroids on brain function: A concept arising from the heterogeneity of brain receptor systems. Psychoneuroendocrinology, 12, 83-105. doi:10.1016/0306-4530(87)90040-0

Dickerson, S. S., \& Kemeny, M. E. (2004). Acute stressors and cortisol responses: A theoretical integration and synthesis of laboratory research. Psychological Bulletin, 130, 355-391. doi:10.1037/ 0033-2909.130.3.355

Dux, P. E., Ivanoff, J., Asplund, C. L., \& Marois, R. (2006). Isolation of a central bottleneck of information processing with time-resolved fMRI. Neuron, 52, 1109-1120. doi:10.1016/j.neuron.2006.11.009

Easterbrook, J. A. (1959). The effect of emotion on cue utilization and the organization of behavior. Psychological Review, 66, 183201. doi: $10.1037 / \mathrm{h} 0047707$

Elzinga, B. M., \& Roelofs, K. (2005). Cortisol-induced impairments of working memory require acute sympathetic activation. Behavioral Neuroscience, 119, 98-103. doi:10.1037/0735-7044.119.1.98
Fischer, R., \& Hommel, B. (2012). Deep thinking increases task-set shielding and reduces shifting flexibility in dual-task performance. Cognition, 123, 303-307. doi:10.1016/j.cognition.2011.11.015

Fischer, R., Miller, J., \& Schubert, T. (2007a). Evidence for parallel semantic memory retrieval in dual tasks. Memory \& Cognition, 35, 1685-1699. doi:10.3758/BF03193502

Fischer, R., Schubert, T., \& Liepelt, R. (2007b). Accessory stimuli modulate effects of nonconscious priming. Perception \& Psychophysics, 69, 9-22. doi:10.3758/BF03194449

Gonzalez-Bono, E., Rohleder, N., Hellhammer, D. H., Salvador, A., \& Kirschbaum, C. (2002). Glucose but not protein or fat load amplifies the cortisol response to psychosocial stress. Hormones and Behavior, 41, 328-333. doi:10.1006/hbeh.2002.1766

Hale, H. B., Ellis, J. P. Jr., \& Kratochvil, C. H. (1959). Effects of piloting supersonic aircraft on plasma corticosteroids and bicarbonate. Journal of Applied Physiology, 14, 629-631.

Het, S., Rohleder, N., Schoofs, D., Kirschbaum, C., \& Wolf, O. T. (2009). Neuroendocrine and psychometric evaluation of a placebo version of the "Trier Social Stress Test". Psychoneuroendocrinology, 34, 1075-1086. doi:10.1016/j.psyneuen.2009.02.008

Hockey, G. R. (1997). Compensatory control in the regulation of human performance under stress and high workload: A cognitive-energetical framework. Biological Psychology, 45, 73-93. doi:10.1016/S0301-0511(96)05223-4

Hommel, B. (1998). Automatic stimulus-response translation in dualtask performance. Journal of Experimental Psychology. Human Perception and Performance, 24, 1368-1384. doi:10.1037/00961523.24.5.1368

Hsu, F. C., Garside, M. J., Massey, A. E., \& McAllister-Williams, R. H. (2003). Effects of a single dose of cortisol on the neural correlates of episodic memory and error processing in healthy volunteers. Psychopharmacology, 167, 431-442. doi:10.1007/s00213-0031413-2

Huguet, P., Galvaing, M. P., Monteil, J. M., \& Dumas, F. (1999). Social presence effects in the Stroop task: Further evidence for an attentional view of social facilitation. Journal of Personality and Social Psychology, 77, 1011-1025. doi:10.1037/0022-3514.77.5.1011

Kahneman, D. (1973). Attention and effort. Englewood Cliffs, NJ: Prentice-Hall.

Keinan, G., Friedland, N., Kahneman, D., \& Roth, D. (1999). The effect of stress on the suppression of erroneous competing responses. Anxiety, Stress, and Coping, 12, 455-476. doi:10.1080/10615809908249321

Kern, S., Oakes, T. R., Stone, C. K., McAuliff, E. M., Kirschbaum, C., \& Davidson, R. J. (2008). Glucose metabolic changes in the prefrontal cortex are associated with HPA axis response to a psychosocial stressor. Psychoneuroendocrinology, 33, 517-529. doi:10.1016/j.psyneuen.2008.01.010

Kirschbaum, C., Gonzalez Bono, E., Rohleder, N., Gessner, C., Pirke, K. M., Salvador, A., \& Hellhammer, D. H. (1997). Effects of fasting and glucose load on free cortisol responses to stress and nicotine. Journal of Clinical Endocrinology and Metabolism, 82, 1101-1105. doi:10.1210/jc.82.4.1101

Kirschbaum, C., \& Hellhammer, D. H. (1994). Salivary cortisol in psychoneuroendocrine research: Recent developments and applications. Psychoneuroendocrinology, 19, 313-333. doi:10.1016/ 0306-4530(94)90013-2

Kirschbaum, C., Kudielka, B. M., Gaab, J., Schommer, N. C., \& Hellhammer, D. H. (1999). Impact of gender, menstrual cycle phase, and oral contraceptives on the activity of the hypothalamus-pituitary-adrenal axis. Psychosomatic Medicine, 61, 154162.

Kirschbaum, C., Pirke, K. M., \& Hellhammer, D. H. (1993). The "Trier Social Stress Test"- A tool for investigating psychobiological stress responses in a laboratory setting. Neuropsychobiology, 28, 76-81. doi:10.1159/000119004 
Koch, I. (2009). The role of crosstalk in dual-task performance: Evidence from manipulating response-code overlap. Psychological Research, 73, 417-424. doi:10.1007/s00426-008-0152-8

Koch, I., Gade, M., Schuch, S., \& Philipp, A. M. (2010). The role of inhibition in task switching: A review. Psychonomic Bulletin \& Review, 17, 1-14. doi:10.3758/PBR.17.1.1

Koechlin, E., Basso, G., Pietrini, P., Panzer, S., \& Grafman, J. (1999). The role of the anterior prefrontal cortex in human cognition. Nature, 399, 148-151. doi:10.1038/20178

Kuhl, J. (1994). Action versus state orientation: Psychometric properties of the action control scale (ACS-90). In J. Kuhl \& J. Beckmann (Eds.), Volition and personality: Action versus state orientation (pp. 47-59). Seattle, WA: Hogrefe.

Kuhl, J. (2000). A functional-design approach to motivation and selfregulation: The dynamics of personality systems interactions. In M. Boekaerts, P. R. Pintrich, \& M. Zeidner (Eds.), Handbook of selfregulation (pp. 111-169). Burlington, MA: Elsevier/Academic Press.

Kuhl, J., \& Beckmann, J. (1994). Volition and personality: Action versus state orientation. Seattle, WA: Hogrefe.

Lehle, C., \& Hübner, R. (2009). Strategic capacity sharing between two tasks: Evidence from tasks with the same and with different task sets. Psychological Research, 73, 707-726. doi:10.1007/ s00426-008-0162-6

Lehle, C., Steinhauser, M., \& Hübner, R. (2009). Serial or parallel processing in dual tasks: What is more effortful? Psychophysiology, 46, 502-509. doi:10.1111/j.1469-8986.2009.00806.x

Lien, M.-C., \& Proctor, R. W. (2002). Stimulus-response compatibility and psychological refractory period effects: Implications for response selection. Psychonomic Bulletin \& Review, 9, 212-238. doi:10.3758/BF03196277

Liston, C., McEwen, B. S., \& Casey, B. J. (2009). Psychosocial stress reversibly disrupts prefrontal processing and attentional control. Proceedings of the National Academy of Sciences, 106, 912-917. doi:10.1073/pnas.0807041106

Logan, G. D., \& Gordon, R. D. (2001). Executive control of visual attention in dual-task situations. Psychological Review, 108, 393 434. doi:10.1037/0033-295X.108.2.393

Logan, G. D., \& Schulkind, M. D. (2000). Parallel memory retrieval in dual-task situations: I. Semantic memory. Journal of Experimental Psychology. Human Perception and Performance, 26, 1072-1090. doi:10.1037/0096-1523.26.3.1072

Lovallo, W. R., Farag, N. H., Vincent, A. S., Thomas, T. L., \& Wilson, M. F. (2006). Cortisol responses to mental stress, exercise, and meals following caffeine intake in men and women. Pharmacology, Biochemistry, and Behavior, 83, 441-447. doi:10.1016/ j.pbb.2006.03.005

Luria, R., \& Meiran, N. (2003). Online order control in the psychological refractory period paradigm. Journal of Experimental Psychology. Human Perception and Performance, 29, 556-574. doi:10.1037/0096-1523.29.3.556

Marois, R., \& Ivanoff, J. (2005). Capacity limits of information processing in the brain. Trends in Cognitive Sciences, 9, 296-305. doi:10.1016/j.tics.2005.04.010

Meyer, D. E., \& Kieras, D. E. (1997). A computational theory of executive cognitive processes and multiple-task performance: Part 1. Basic mechanisms. Psychological Review, 104, 3-65. doi:10.1037/0033-295X.104.1.3

Miller, J. (2006). Backward crosstalk effects in psychological refractory period paradigms: Effects of second-task response types on first-task response latencies. Psychological Research, 70, 484-493. doi: $10.1007 / \mathrm{s} 00426-005-0011-9$

Miller, J., Ulrich, R., \& Rolke, B. (2009). On the optimality of serial and parallel processing in the psychological refractory period paradigm: Effects of the distribution of stimulus onset asynchronies. Cognitive Psychology, 58, 273-310. doi:10.1016/j.cogpsych.2006.08.003
Nater, U. M., \& Rohleder, N. (2009). Salivary alpha-amylase as a noninvasive biomarker for the sympathetic nervous system: Current state of research. Psychoneuroendocrinology, 34, 486-496. doi:10.1016/j.psyneuen.2009.01.014

Navon, D., \& Miller, J. (1987). Role of outcome conflict in dualtask interference. Journal of Experimental Psychology. Human Perception and Performance, 13, 435-448. doi:10.1037/00961523.13.3.435

Navon, D., \& Miller, J. (2002). Queuing or sharing? A critical evaluation of the single-bottleneck notion. Cognitive Psychology, 44, 193-251. doi:10.1006/cogp.2001.0767

Oei, N. Y., Everaerd, W. T., Elzinga, B. M., van Well, S., \& Bermond, B. (2006). Psychosocial stress impairs working memory at high loads: An association with cortisol levels and memory retrieval. Stress, 9, 133-141. doi:10.1080/10253890600965773

Oriet, C., Tombu, M., \& Jolicœur, P. (2005). Symbolic distance affects two processing loci in the number comparison task. Memory \& Cognition, 33, 913-926. doi:10.3758/BF03193085

Pashler, H. (1994). Graded capacity-sharing in dual-task interference? Journal of Experimental Psychology. Human Perception and Performance, 20, 330-342. doi:10.1037/0096-1523.20.2.330

Pashler, H. E. (1998). The psychology of attention. Cambridge, MA: MIT Press.

Perlman, W. R., Webster, M. J., Herman, M. M., Kleinman, J. E., \& Weickert, C. S. (2007). Age-related differences in glucocorticoid receptor mRNA levels in the human brain. Neurobiology of Aging, 28, 447-458. doi:10.1016/j.neurobiolaging.2006.01.010

Petzold, A., Plessow, F., Goschke, T., \& Kirschbaum, C. (2010). Stress reduces use of negative feedback in a feedback-based learning task. Behavioral Neuroscience, 124, 248-255. doi:10.1037/ a 0018930

Pincus, G., \& Hoagland, H. (1943). Steroid excretion and the stress of flying. The Journal of Aviation Medicine, 14, 173-182.

Plessow, F., Fischer, R., Kirschbaum, C., \& Goschke, T. (2011). Inflexibly focused under stress: Acute psychosocial stress increases shielding of action goals at the expense of reduced cognitive flexibility with increasing time lag to the stressor. Journal of Cognitive Neuroscience, 23, 3218-3227. doi:10.1162/ jocn a 00024

Plessow, F., Kiesel, A., \& Kirschbaum, C. (2012). The stressed prefrontal cortex and goal-directed behaviour: Acute psychosocial stress impairs the flexible implementation of task goals. Experimental Brain Research, 216, 397-408. doi:10.1007/s00221-0112943-1

Prüssner, J. C., Kirschbaum, C., Meinlschmid, G., \& Hellhammer, D. H. (2003). Two formulas for computation of the area under the curve represent measures of total hormone concentration versus time-dependent change. Psychoneuroendocrinology, 28, 916931. doi:10.1016/S0306-4530(02)00108-7

Qin, S., Hermans, E. J., van Marle, H. J., Luo, J., \& Fernandez, G. (2009). Acute psychological stress reduces working memoryrelated activity in the dorsolateral prefrontal cortex. Biological Psychiatry, 66, 25-32. doi:10.1016/j.biopsych.2009.03.006

Ramos, B. P., \& Arnsten, A. F. (2007). Adrenergic pharmacology and cognition: Focus on the prefrontal cortex. Pharmacology \& Therapeutics, 113, 523-536. doi:10.1016/j.pharmthera.2006.11.006

Rohleder, N., \& Kirschbaum, C. (2006). The hypothalamic-pituitaryadrenal (HPA) axis in habitual smokers. International Journal of Psychophysiology, 59, 236-243. doi:10.1016/j.ijpsycho.2005.10.012

Rohleder, N., \& Nater, U. M. (2009). Determinants of salivary alphaamylase in humans and methodological considerations. Psychoneuroendocrinology, 34, 469-485. doi:10.1016/j.psyneuen.2008.12.004

Sanchez, M. M., Young, L. J., Plotsky, P. M., \& Insel, T. R. (2000). Distribution of corticosteroid receptors in the rhesus brain: Relative absence of glucocorticoid receptors in the hippocampal formation. Journal of Neuroscience, 20, 4657-4668. 
Scholz, U., La Marca, R., Nater, U. M., Aberle, I., Ehlert, U., Hornung, R., Martin, M., \& Kliegel, M. (2009). Go no-go performance under psychosocial stress: Beneficial effects of implementation intentions. Neurobiology of Learning and Memory, 91, 89-92. doi:10.1016/j.nlm.2008.09.002

Schoofs, D., Wolf, O. T., \& Smeets, T. (2009). Cold pressor stress impairs performance on working memory tasks requiring executive functions in healthy young men. Behavioral Neuroscience, 123, 1066-1075. doi:10.1037/a0016980

Schubert, T., Fischer, R., \& Stelzel, C. (2008). Response activation in overlapping tasks and the response-selection bottleneck. Journal of Experimental Psychology. Human Perception and Performance, 34, 376-397. doi:10.1037/0096-1523.34.2.376

Schuch, S., \& Koch, I. (2004). The costs of changing the representation of action: Response repetition and response-response compatibility in dual tasks. Journal of Experimental Psychology. Human Perception and Performance, 30, 566-582. doi:10.1037/00961523.30.3.566

Schwabe, L., Wolf, O. T., \& Oitzl, M. S. (2010). Memory formation under stress: Quantity and quality. Neuroscience and Biobehavioral Reviews, 34, 584-591. doi:10.1016/j.neubiorev.2009.11.015

Sigman, M., \& Dehaene, S. (2006). Dynamics of the central bottleneck: Dual-task and task uncertainty. PLoS Biology, 4, e220. doi:10.1371/journal.pbio. 0040220
Smeets, T., Dziobek, I., \& Wolf, O. T. (2009). Social cognition under stress: Differential effects of stress-induced cortisol elevations in healthy young men and women. Hormones and Behavior, 55, 507-513. doi:10.1016/j.yhbeh.2009.01.011

Stelzel, C., Brandt, S. A., \& Schubert, T. (2009). Neural mechanisms of concurrent stimulus processing in dual tasks. NeuroImage, 48, 237-248. doi:10.1016/j.neuroimage.2009.06.064

Steyer, R., Schwenkmezger, P., Notz, P., \& Eid, M. (1997). Der mehrdimensionale Befindlichkeitsfragebogen (MDBF) [The multidimensional mental-state questionnaire $(M D B F)]$. Göttingen: Hogrefe.

Tombu, M., \& Jolicœur, P. (2003). A central capacity sharing model of dual-task performance. Journal of Experimental Psychology. Human Perception and Performance, 29, 3-18. doi:10.1037/00961523.29.1.3

Vijayraghavan, S., Wang, M., Birnbaum, S. G., Williams, G. V., \& Arnsten, A. F. (2007). Inverted-U dopamine D1 receptor actions on prefrontal neurons engaged in working memory. Nature Neuroscience, 10, 376-384. doi: $10.1038 / \mathrm{nn} 1846$

Wolf, O. T., Schommer, N. C., Hellhammer, D. H., McEwen, B. S., \& Kirschbaum, C. (2001). The relationship between stress induced cortisol levels and memory differs between men and women. Psychoneuroendocrinology, 26, 711-720. doi:10.1016/S03064530(01)00025-7 